\title{
Limits on the High-Energy Gamma and Neutrino Fluxes from the SGR 1806-20 Giant Flare of 27 December 2004 with the AMANDA-II Detector
}

A. Achterberg, ${ }^{30}$ M. Ackermann, ${ }^{32} \mathrm{~J}$. Adams, ${ }^{10} \mathrm{~J}$. Ahrens,${ }^{20} \mathrm{~K}$. Andeen, ${ }^{19}$ D. W. Atlee,${ }^{28} \mathrm{~J} . \mathrm{N}$. Bahcall, ${ }^{24, *} \mathrm{X}$. Bai, ${ }^{22}$ B. Baret, ${ }^{8}$ M. Bartelt, ${ }^{12}$ S. W. Barwick, ${ }^{15}$ R. Bay,${ }^{4}$ K. Beattie, ${ }^{6}$ T. Becka,${ }^{20}$ J. K. Becker, ${ }^{12}$ K.-H. Becker, ${ }^{31}$ P. Berghaus ${ }^{7}$ D. Berley, ${ }^{11}$ E. Bernardini, ${ }^{32}$ D. Bertrand, ${ }^{7}$ D. Z. Besson, ${ }^{16}$ E. Blaufuss, ${ }^{11}$ D. J. Boersma, ${ }^{19}$ C. Bohm ${ }^{26}$ J. Bolmont, ${ }^{32}$ S. Böser ${ }^{32}$ O. Botner ${ }^{29}$ A. Bouchta, ${ }^{29}$ J. Braun, ${ }^{19}$ C. Burgess,${ }^{26}$ T. Burgess,${ }^{26}$ T. Castermans, ${ }^{21}$ D. Chirkin, ${ }^{6}$ B. Christy, ${ }^{11}$ J. Clem, ${ }^{22}$ D. F. Cowen, ${ }^{28,27}$ M. V. D'Agostino, ${ }^{4}$ A. Davour, ${ }^{29}$ C. T. Day,${ }^{6}$ C. De Clercq,${ }^{8}$ L. Demirörs, ${ }^{22}$ F. Descamps,${ }^{13}$ P. Desiati, ${ }^{19}$ T. DeYoung, ${ }^{28}$ J. C. Diaz-Velez, ${ }^{19}$ J. Dreyer, ${ }^{12}$ J. P. Dumm, ${ }^{19}$ M. R. Duvoort,${ }^{30}$ W. R. Edwards, ${ }^{6}$ R. Ehrlich, ${ }^{11}$ J. Eisch, ${ }^{25}$ R. W. Ellsworth, ${ }^{11}$ P. A. Evenson, ${ }^{22}$ O. Fadiran, ${ }^{2}$ A. R. Fazely, ${ }^{3}$ T. Feser ${ }^{20}$ K. Filimonov, ${ }^{4}$ B. D. Fox ${ }^{28}$ T. K. Gaisser, ${ }^{22}$ J. Gallagher, ${ }^{18}$ R. Ganugapati, ${ }^{19}$ H. Geenen, ${ }^{31}$ L. Gerhardt, ${ }^{15}$ A. Goldschmidt, ${ }^{6}$ J. A. Goodman, ${ }^{11}$ R. Gozzini, ${ }^{20}$ S. Grullon, ${ }^{19}$ A. Groß,${ }^{14}$ R. M. Gunasingha, ${ }^{3}$ M. Gurtner, ${ }^{31}$ A. Hallgren, ${ }^{29}$ F. Halzen, ${ }^{19}$ K. Han, ${ }^{10}$ K. Hanson, ${ }^{19}$ D. Hardtke, ${ }^{4}$ R. Hardtke, ${ }^{25}$ T. Harenberg, ${ }^{31}$ J. E. Hart,${ }^{28}$ T. Hauschildt,${ }^{22}$ D. Hays, ${ }^{6}$ J. Heise,${ }^{30}$ K. Helbing,${ }^{31}$ M. Hellwig, ${ }^{20}$ P. Herquet, ${ }^{21}$ G. C. Hill, ${ }^{19}$ J. Hodges, ${ }^{19}$ K. D. Hoffman, ${ }^{11}$ B. Hommez, ${ }^{13}$ K. Hoshina, ${ }^{19}$ D. Hubert, ${ }^{8}$ B. Hughey ${ }^{19}$ P. O. Hulth ${ }^{26}$ K. Hultqvist ${ }^{26}{ }^{26}$ S. Hundertmark, ${ }^{26}$ J.-P. Hülß, ${ }^{31}$ A. Ishihara, ${ }^{19}$ J. Jacobsen, ${ }^{6}$ G. S. Japaridze, ${ }^{2}$ A. Jones, ${ }^{6}$ J. M. Joseph, ${ }^{6}$ K.-H. Kampert, ${ }^{31}$ A. Karle, ${ }^{19}$ H. Kawai, ${ }^{9}$ J. L. Kelley, ${ }^{19}$ M. Kestel, ${ }^{28}$ N. Kitamura ${ }^{19}$ S. R. Klein, ${ }^{6}$ S. Klepser, ${ }^{32}$ G. Kohnen, ${ }^{21}$ H. Kolanoski, ${ }^{5}$ L. Köpke, ${ }^{20}$ M. Krasberg, ${ }^{19}$ K. Kuehn, ${ }^{15}$ H. Landsman, ${ }^{19}$ H. Leich, ${ }^{32}$ I. Liubarsky, ${ }^{17}$ J. Lundberg, ${ }^{29}$ J. Madsen, ${ }^{25}$ K. Mase, ${ }^{9}$ H. S. Matis, ${ }^{6}$ T. McCauley, ${ }^{6}$ C. P. McParland, ${ }^{6}$ A. Meli, ${ }^{12}$ T. Messarius, ${ }^{12}$ P. Mészáros,${ }^{27,28}$ H. Miyamoto, ${ }^{9}$ A. Mokhtarani, ${ }^{6}$ T. Montaruli, ${ }^{19, \dagger}$ A. Morey, ${ }^{4}$ R. Morse,${ }^{19}$ S. M. Movit ${ }^{27}$ K. Münich, ${ }^{12}$ R. Nahnhauer, ${ }^{32}$ J. W. Nam, ${ }^{15}$ P. Nießen, ${ }^{22}$ D. R. Nygren, ${ }^{6}$ H. Ögelman, ${ }^{19}$ Ph. Olbrechts, ${ }^{8}$ A. Olivas, ${ }^{11}$ S. Patton, ${ }^{6}$ C. Peña-Garay, ${ }^{24}$ C. Pérez de los Heros,${ }^{29}$ A. Piegsa, ${ }^{20}$ D. Pieloth,${ }^{32}$ A. C. Pohl,${ }^{29,5}$ R. Porrata, ${ }^{4}$ J. Pretz, ${ }^{11}$ P. B. Price, ${ }^{4}$ G. T. Przybylski, ${ }^{6}$ K. Rawlins,${ }^{1}$ S. Razzaque,${ }^{27,28}$ F. Refflinghaus,${ }^{12}$ E. Resconi,${ }^{14}$ W. Rhode,${ }^{12}$ M. Ribordy, ${ }^{21}$ A. Rizzo,${ }^{8}$ S. Robbins, ${ }^{31}$ P. Roth,,${ }^{11}$ C. Rott, ${ }^{28}$ D. Rutledge, ${ }^{28}$ D. Ryckbosch,${ }^{13}$ H.-G. Sander, ${ }^{20}$ S. Sarkar,${ }^{23}$ S. Schlenstedt,${ }^{32}$ T. Schmidt, ${ }^{11}$ D. Schneider, ${ }^{19}$ D. Seckel, ${ }^{22}$ S. H. Seo, ${ }^{28}$ S. Seunarine, ${ }^{10}$ A. Silvestri, ${ }^{15}$ A. J. Smith, ${ }^{11}$ M. Solarz, ${ }^{4}$ C. Song, ${ }^{19}$ J. E. Sopher, ${ }^{6}$ G. M. Spiczak, ${ }^{25}$ C. Spiering, ${ }^{32}$ M. Stamatikos, ${ }^{19}$ T. Stanev,${ }^{22}$ P. Steffen, ${ }^{32}$ T. Stezelberger, ${ }^{6}$ R. G. Stokstad, ${ }^{6}$ M. C. Stoufer, ${ }^{6}$ S. Stoyanov, ${ }^{22}$ E. A. Strahler, ${ }^{19}$ T. Straszheim, ${ }^{11}$ K.-H. Sulanke, ${ }^{32}$ G. W. Sullivan, ${ }^{11}$ T. J. Sumner, ${ }^{17}$ I. Taboada, ${ }^{4}$ O. Tarasova, ${ }^{32}$ A. Tepe,${ }^{31}$ L. Thollander, ${ }^{26}$ S. Tilav, ${ }^{22}$ P. A. Toale,${ }^{28}$ D. Turčan, ${ }^{11}$ N. van Eijndhoven, ${ }^{30}$ J. Vandenbroucke, ${ }^{4}$ A. Van Overloop,${ }^{13}$ B. Voigt, ${ }^{32}$ W. Wagner, ${ }^{28}$ C. Walck, ${ }^{26}$ H. Waldmann, ${ }^{32}$ M. Walter, ${ }^{32}$ Y.-R. Wang, ${ }^{19}$ C. Wendt, ${ }^{19}$ C. H. Wiebusch, ${ }^{31}$ G. Wikström, ${ }^{26}$ D. R. Williams, ${ }^{28}$ R. Wischnewski, ${ }^{32}$ H. Wissing, ${ }^{32}$ K. Woschnagg, ${ }^{4}$ X. W. Xu, ${ }^{3}$ G. Yodh,${ }^{15}$ S. Yoshida, ${ }^{9}$ and J. D. Zornoza ${ }^{19, \S}$

(IceCube Collaboration)

\footnotetext{
${ }^{1}$ Department of Physics and Astronomy, University of Alaska, Anchorage, 3211 Providence Drive, Anchorage, Alaska 99508, USA ${ }^{2}$ CTSPS, Clark-Atlanta University, Atlanta, Georgia 30314, USA

${ }^{3}$ Department of Physics, Southern University, Baton Rouge, Louisiana 70813, USA

${ }^{4}$ Department of Physics, University of California, Berkeley, California 94720, USA

${ }^{5}$ Institut für Physik, Humboldt Universität zu Berlin, D-12489 Berlin, Germany

${ }^{6}$ Lawrence Berkeley National Laboratory, Berkeley, California 94720, USA

${ }^{7}$ Science Faculty, Université Libre de Bruxelles, CP230, B-1050 Brussels, Belgium

${ }^{8}$ Vrije Universiteit Brussel, Dienst ELEM, B-1050 Brussels, Belgium

${ }^{9}$ Department of Physics, Chiba University, Chiba 263-8522, Japan

${ }^{10}$ Department of Physics and Astronomy, University of Canterbury, Private Bag 4800, Christchurch, New Zealand

${ }^{11}$ Department of Physics, University of Maryland, College Park, Maryland 20742, USA

${ }^{12}$ Department of Physics, Universität Dortmund, D-44221 Dortmund, Germany

${ }^{13}$ Department of Subatomic and Radiation Physics, University of Gent, B-9000 Gent, Belgium

${ }^{14}$ Max-Planck-Institut für Kernphysik, D-69177 Heidelberg, Germany

${ }^{15}$ Department of Physics and Astronomy, University of California, Irvine, California 92697, USA

${ }^{16}$ Department of Physics and Astronomy, University of Kansas, Lawrence, Kansas 66045, USA

${ }^{17}$ Blackett Laboratory, Imperial College, London SW7 2BW, United Kingdom

${ }^{18}$ Department of Astronomy, University of Wisconsin, Madison, Wisconsin 53706, USA

${ }^{19}$ Department of Physics, University of Wisconsin, Madison, Wisconsin 53706, USA

${ }^{20}$ Institute of Physics, University of Mainz, Staudinger Weg 7, D-55099 Mainz, Germany

${ }^{21}$ University of Mons-Hainaut, 7000 Mons, Belgium
} 


\author{
${ }^{22}$ Bartol Research Institute, University of Delaware, Newark, Delaware 19716, USA \\ ${ }^{23}$ Department of Physics, University of Oxford, 1 Keble Road, Oxford OX1 3NP, United Kingdom \\ ${ }^{24}$ Institute for Advanced Study, Princeton, New Jersey 08540, USA \\ ${ }^{25}$ Department of Physics, University of Wisconsin, River Falls, Wisconsin 54022, USA \\ ${ }^{26}$ Department of Physics, Stockholm University, SE-10691 Stockholm, Sweden \\ ${ }^{27}$ Department of Astronomy and Astrophysics, Pennsylvania State University, University Park, Pennsylvania 16802, USA \\ ${ }^{28}$ Department of Physics, Pennsylvania State University, University Park, Pennsylvania 16802, USA \\ ${ }^{29}$ Division of High Energy Physics, Uppsala University, S-75121 Uppsala, Sweden \\ ${ }^{30}$ Department of Physics and Astronomy, Utrecht University/SRON, NL-3584 CC Utrecht, The Netherlands \\ ${ }^{31}$ Department of Physics, University of Wuppertal, D-42119 Wuppertal, Germany \\ ${ }^{32}$ DESY, D-15735 Zeuthen, Germany
}

(Received 11 July 2006; published 28 November 2006)

On 27 December 2004, a giant $\gamma$ flare from the Soft Gamma-Ray Repeater 1806-20 saturated many satellite gamma-ray detectors, being the brightest transient event ever observed in the Galaxy. AMANDAII was used to search for down-going muons indicative of high-energy gammas and/or neutrinos from this object. The data revealed no significant signal, so upper limits (at 90\% C.L.) on the normalization constant were set: $0.05(0.5) \mathrm{TeV}^{-1} \mathrm{~m}^{-2} \mathrm{~s}^{-1}$ for $\gamma=-1.47(-2)$ in the gamma flux and $0.4(6.1) \mathrm{TeV}^{-1} \mathrm{~m}^{-2} \mathrm{~s}^{-1}$ for $\gamma=-1.47(-2)$ in the high-energy neutrino flux.

DOI: 10.1103/PhysRevLett.97.221101

PACS numbers: 95.85.Pw, 95.55.Ka, 95.55.Vj, 97.60.Gb

Introduction. - Soft gamma-ray repeaters (SGRs) are $\mathrm{x}$ ray pulsars which have quiescent soft $(2-10 \mathrm{keV})$ periodic $\mathrm{x}$-ray emissions with periods ranging from 5 to $10 \mathrm{~s}$ and luminosities of the order of $10^{33-35} \mathrm{erg} / \mathrm{s}$. They exhibit repetitive bursts lasting $\sim 0.1 \mathrm{~s}$ which reach peak luminosities of $\sim 10^{41} \mathrm{erg} / \mathrm{s}$ in $\mathrm{x}$ rays and $\gamma$ rays. There are four known SGRs, three in the Milky Way (including SGR 1860-20) and one in the Large Magellanic Cloud. Three of the four known SGRs have had hard spectrum $(\sim \mathrm{MeV}$ energy) giant flares with luminosities reaching up to $\sim 10^{47} \mathrm{erg} / \mathrm{s}$. The first of these giant flares (from SGR 0525-66 [1]) was observed on 5 March 1979 by the Venera 11 and 12 spacecraft. SGR $1900+14$ exhibited a giant flare in 1998 [1]. The most recent and brightest flare came from SGR 1806-20 on 27 December 2004. This flare lasted about 5 min (the duration of the initial spike was $\sim 0.2 \mathrm{~s}$ ) and had a peak luminosity of $\sim 2 \times 10^{47} \mathrm{erg} / \mathrm{s}$ and a total energy emission of $\sim 5 \times 10^{46}$ erg [2], which made it the most luminous transient event ever observed in the Galaxy. This flare was observed by several satellite experiments [3-5], although they saturated during the blast. Recent estimates locate the source at a distance of $15.1_{-1.3}^{+1.8} \mathrm{kpc}$ [6], but this value is still under debate [7].

The favored "magnetar" model for these objects is a neutron star with a huge magnetic field $\left(B \sim 10^{15} \mathrm{G}\right)$. These giant flares can be explained as global crustal fractures due to magnetic field rearrangements liberating a high flux of $\mathrm{x}$ rays and $\gamma$ rays [8].

The uncertainties in the spectral measurement are large, but fits to the data favor the presence of a nonthermal component [2,9], which would imply an important highenergy emission. Indeed, SGRs have been proposed as the sources of the ultra-high-energy cosmic rays [10].

The possibility of using underground detectors to observe the muons produced in the electromagnetic showers induced by $\mathrm{TeV}$ gammas generated in these flares was presented in Ref. [11]. There have also been suggestions of high-energy neutrino production. Gelfand et al. [12] interpret the observations of an expanding radio source [13] as the hint of a baryonic fireball. Ioka et al. [14] also argue that high-energy neutrino production can be related to the fraction of burst energy released in the form of baryons.

The December 2004 giant flare represents an excellent opportunity to probe the high-energy spectrum of these sources by looking for events correlated in time and space with this flare. In this Letter we present the results of a search for a gamma and/or a neutrino signal during the SGR 1806-20 giant flare using data from the AMANDA-II detector. The short duration of these events and the fact that they come from a pointlike source result in negligible atmospheric muon and neutrino backgrounds. For the first time in AMANDA (see [15] for an example of a previous search in other kinds of sources), we used down-going muons to look for muon photoproduction in the atmosphere, already proposed in Ref. [16].

AMANDA is currently running in its AMANDA-II configuration of a 3D array of 677 optical modules (OMs) distributed along 19 strings deployed at depths of 1500$2000 \mathrm{~m}$ in the South Pole ice [17]. These 8 in. photomultipliers, enclosed in pressure-resistant glass spheres, make it possible to reconstruct the direction and energy of relativistic muons through timing and intensity of their Cherenkov light. The ice layer above the detector reduces the background of atmospheric muons by more than 5 orders of magnitude compared to the surface flux.

Analysis technique.-For events like the burst of the SGR 1806-20 in December 2004, in which most of the energy emitted during the flare is concentrated in a $1 \mathrm{~s} \mathrm{time}$ scale, the precise time and location of the event imply that 
the background of atmospheric muons becomes negligible [11], thereby allowing a search for $\mathrm{TeV} \gamma$ rays and downgoing $\mathrm{TeV}$ neutrinos.

The IceCube collaboration, which operates the AMANDA detector, follows a policy of blindness in its analysis strategies. By studying the expected backgrounds and signals prior to looking at the data, the analysis can be designed in an unbiased fashion. In the case of expected small signals, this is particularly relevant for having a clear procedure to determine the probability of an event to be produced by background. Thus, in this analysis, the determination of the optimum selection criteria is done using the simulation of the signal and comparing it with the expected background. The procedure, similar to that used in the search for upward moving neutrinos from gammaray bursts [18], is as follows: (i) The background on source and off time is calculated using real data, keeping blind 10 min around the burst onset. About $1 \mathrm{~d}$ of off-time data was used to monitor the stability of the detector. (ii) The signal from the source is simulated in order to estimate the angular resolution and the effective area of the detector. (iii) The appropriate time window is estimated, based on the flare onset times given by different $\mathrm{x}$-ray satellites and their counting rates. (iv) The optimum search bin size is found by minimizing the model discovery factor (MDF) [19], defined as

$$
\mathrm{MDF}=\frac{\mu\left(n_{b}, \text { C.L., SP }\right)}{n_{s}}
$$

where $\mu$ is the Poisson mean of the number of signal events which would result in rejection of the background hypothesis, at the chosen C.L., in statistical power (SP) percent of equivalent measurements, and $n_{s}$ is the number of signal events predicted by the model. This definition is analogous to the model rejection factor (MRF) (see Ref. [19]) that is used for setting upper limits. In the case of the MDF, the bin size is optimized to maximize the probability of discovery (for C.L. corresponding to five sigma and SP = $90 \%$ ). (v) Once the optimum search bin size has been found, the unblinding of the data is done; i.e., the events inside the time window and the search bin are counted. (vi) This number of events is translated into a flux, or a flux limit if no significant excess is found through the knowledge of the expected signal in the detector for the given analysis cuts.

Although both $\mathrm{TeV} \gamma$ rays and neutrinos produce muons in the detector array, the optimal choice of selection criteria depends on the assumed signal. The analysis was optimized to the $\mathrm{TeV} \gamma$ signal. Any further optimization for a neutrino signal would be more than offset by the penalty for an additional trials factor.

Data and simulation. - In order to have a background estimate for the flare, a time and angular window have to be defined around the flare at equatorial coordinates (J2000): right ascension $=$ UT 18:08:39.34; declination $=-20^{\circ}$ $24^{\prime} 39.7^{\prime \prime}[13,20,21]$. The bulk of the flare energy was concentrated in less than $0.6 \mathrm{~s}$. The counting rate of the Burst Alert Telescope on board the Swift spacecraft drops by more than 2 orders of magnitudes after $0.6 \mathrm{~s}$ from the onset of the burst [9]. Based on the observation time for each satellite [3,5,22-24], and accounting for their positions, the expected signal times in AMANDA were calculated. The spread of the resulting times indicate that a safe window is $1.5 \mathrm{~s}$, centered at UT 21:20:26.6 of 27 December 2004, the onset time of the flare.

To evaluate the performance of the detector, simulations were performed for several input signals. The CORSIKAQGSJET01 [25] and ANIS [26] codes were used to simulate the photon (and proton) and neutrino interactions, respectively. The range of generated energies for photons was 10 to $10^{5} \mathrm{TeV}$ and for protons was $10 \mathrm{GeV}$ to $10^{5} \mathrm{TeV}$. The tracks were reconstructed with the same iterative loglikelihood fitting procedure that was applied to the real data.

The angular resolution for different cuts has been studied using the simulation of down-going muons generated by cosmic rays. The angular resolution, defined as the median of the angular difference between the true and the reconstructed track, is $3.5^{\circ}$. This value was obtained using atmospheric down-going muon high statistics simulations, and it was checked that this result is robust within $0.1^{\circ}$ for the expected signals of photons and neutrino induced muons. We also considered a variety of spectral indices for the $\gamma$-ray spectrum assuming values given in Ref. [11], and we found that the angular resolution is almost independent of the spectral index. The effective areas, defined as the equivalent area for a perfect detector that is able to detect particles with 100\% efficiency, for gammas and neutrinos are shown in Fig. 1 as a function of the energy.

As noted earlier, the optimum angular window is determined by minimizing the MDF. In Fig. 2 we show the

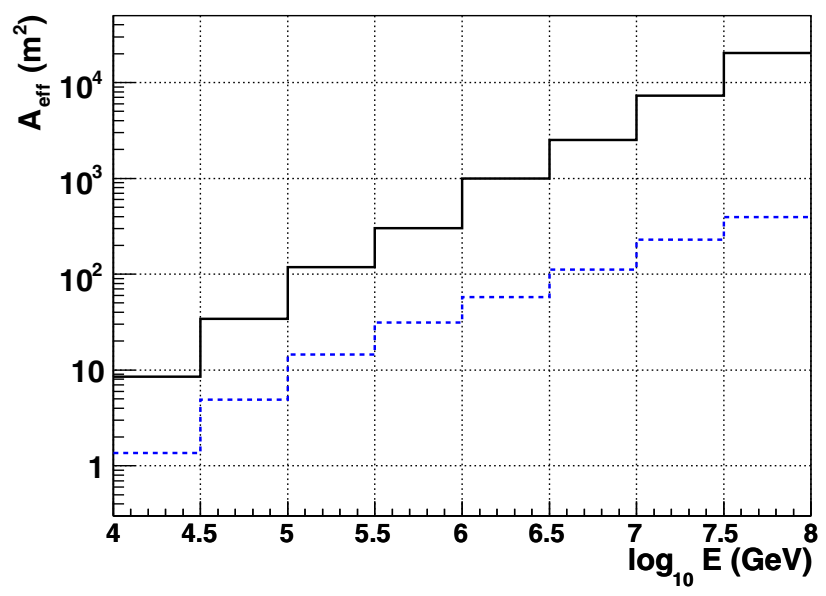

FIG. 1 (color online). Effective area after reconstruction and track quality selection for gammas (solid black line) and neutrinos (dashed blue line). 


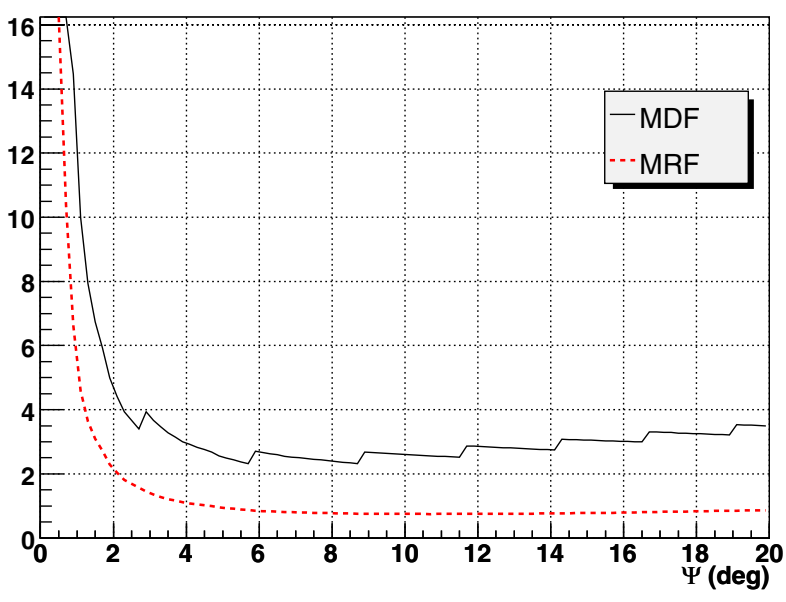

FIG. 2 (color online). Model discovery factor (solid black line) and model rejection factor (dashed red line) as a function of the radius of a circular angular acceptance window. The curves here are for a $E^{-1.47}$ spectrum.

dependence of the MDF and MRF on the radius of a circular angular window around the source position. The steps in the MDF curve are due to the discreteness of the Poisson distribution. It can also be seen that the MRF minimum interval is quite broad, indicating that this variable is not very sensitive to the increase in the number of background events with the increase of the angular window. This is due to the small value of the background in the allowed time window.

The search bin size which optimizes the probability of discovery is at $5.8^{\circ}$. With this cut and a $1.5 \mathrm{~s}$ time window, the expected background is 0.06 counts. For events that satisfy the detector trigger, we keep almost $80 \%$ of the signal in this angular window.

Systematic uncertainties. - Several sources of systematic uncertainties have been considered in this analysis. The uncertainty of the hadronic model calculation is estimated to about $15 \%$. We have compared the muon yields using two different models - CORSIKA/QGSJET and an analytic calculation [16] - and found that they agreed to within $5 \%$. The uncertainty in the detector efficiency (20\%) comes mainly from the overall sensitivity of the OMs and the optical properties of the ice. The effect has been estimated simulating different reasonable ice models and $\mathrm{OM}$ sensitivities.

The stability of the run was checked in order to exclude possible nonparticle events induced by detector electronics. These events are identified by a specific method looking for anomalous values in a set of defined variables. A correction is made for the electronics dead time (17\%, which is a typical value in normal runs). Finally, the simulated and measured distributions of an extensive set of variables, like zenith angle, number of hit optical modules, and hit times, were compared in the search for possible anomalies. In all the cases the agreement was within the systematic errors estimated above.
Results. - Once the optimum search bin size of $5.8^{\circ}$ around the source was determined, we unblinded the $1.5 \mathrm{~s}$ data around the burst looking for events satisfying the analysis requirements. No event was observed in the on-source, on-time window. Then we determined the upper limits [27] of the normalization constant $A_{90}$ at a C.L. of $90 \%$ assuming a power-law energy spectrum,

$$
\frac{d N}{d E}<A_{90}(E / \mathrm{TeV})^{\gamma}
$$

with a cutoff at $10^{5} \mathrm{TeV}$. These limits are shown in Fig. 3 together with the sensitivity of the detector. The effect of the attenuation of the gamma flux by the cosmic microwave background and the Galactic interstellar radiation field has been also taken into account and has been calculated from the results of Ref. [28].

To give an idea of the impact of these limits on theoretical estimates such as the $\gamma$ flux extrapolations presented in Ref. [11], for spectral index $-1.47(-2)$ the limit on the gamma flux normalization constant is $A_{90}=$ $0.05(0.5) \mathrm{TeV}^{-1} \mathrm{~m}^{-2} \mathrm{~s}^{-1}$. The calculation of this limit with the $500 \mathrm{TeV}$ cutoff used in [11] would give 3.3(33) $\mathrm{TeV}^{-1} \mathrm{~m}^{-2} \mathrm{~s}^{-1}$, which rule out spectral indices $\gamma \sim-1.5$, but not softer.

Since the source is above the horizon (hence there is not much column depth for neutrinos to interact), the neutrino flux limits are an order of magnitude worse than the $\mathrm{TeV} \gamma$ limits, but can still be used to constrain models. In cases where there is large baryonic outflow, high-energy neutrinos are produced and the baryons may make the source partially opaque to high-energy photons. Comparing the extrapolations in Ref. [11], for spectral index $-1.47(-2)$ the limit on the $\nu_{\mu}$ flux normalization is

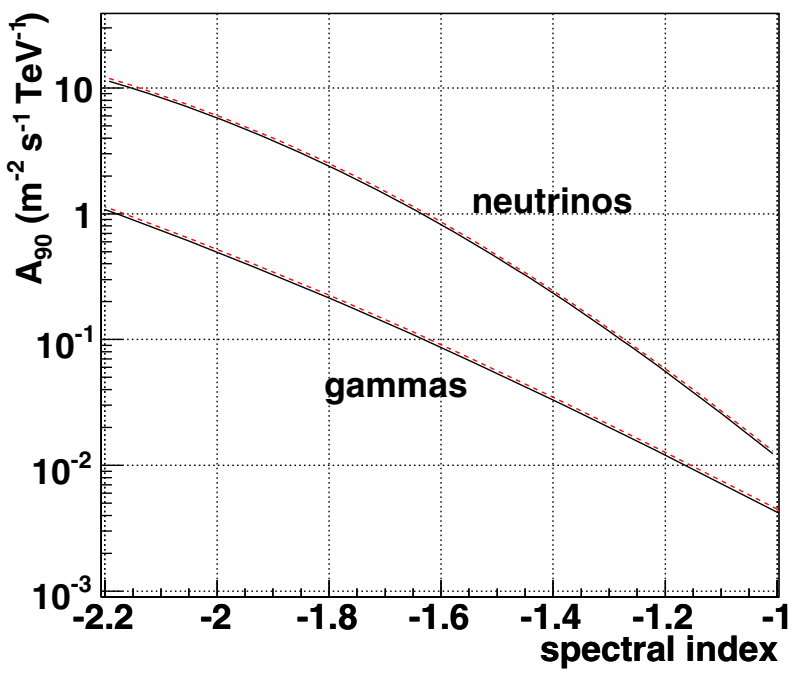

FIG. 3 (color online). Sensitivity (dashed red lines) and limit (solid black lines) to the normalization constant in the flux of gammas (lower line) and neutrinos (upper line), as a function of the spectral index, assuming a flux $\phi(E)=A(E / \mathrm{TeV})^{\gamma}$. 
$A_{90}=0.4(6.1) \mathrm{TeV}^{-1} \mathrm{~m}^{-2} \mathrm{~s}^{-1}$ while the model predicts (accounting for oscillations) $1.7\left(4.1 \times 10^{-4}\right)$ in the same units. We are thus able to exclude an extremely hard neutrino spectrum extrapolated from the measured $\mathrm{MeV}$ photon flux. On the other hand, our limit on the highenergy neutrino fluence is still at least 1 order of magnitude larger than the fluence predicted in Ref. [12].

Conclusions. - In summary, we have searched for $\mathrm{TeV}$ gammas and neutrinos associated with the 27 December 2004 giant flare from SGR 1806-20. We have demonstrated that the background in underground neutrino arrays such as AMANDA or IceCube is low enough so that they can be used as $\mathrm{TeV} \gamma$ detectors for transient events. This is an important result since these detectors offer a large duty cycle and a wide field of view. This analysis of AMANDA data yielded no muons coincident with the flare. We used this muon nonobservation to place stringent limits on $\mathrm{TeV}$ radiation from this source, ruling out some hypothesis proposed in previous papers.

We acknowledge the support from the following agencies: Office of Polar Program and Physics Division of the National Science Foundation, University of Wisconsin Alumni Research Foundation, Department of Energy, and National Energy Research Scientific Computing Center (supported by the Office of Energy Research of the Department of Energy), the NSF-supported TeraGrid system at the San Diego Supercomputer Center (SDSC), and the National Center for Supercomputing Applications (NCSA); Swedish Research Council, Swedish Polar Research Secretariat, and Knut and Alice Wallenberg Foundation, Sweden; German Ministry for Education and Research, Deutsche Forschungsgemeinschaft (DFG), Germany; Fund for Scientific Research (FNRS-FWO), Flanders Institute to Encourage Scientific and Technological Research in Industry (IWT), Belgian Federal Office for Scientific, Technical and Cultural affairs (OSTC); and the Netherlands Organization for Scientific Research (NWO). M. R. acknowledges the support of the SNF (Switzerland). J. D. Z. acknowledges the Marie Curie OIF Program (Contract No. 007921).

\footnotetext{
*Deceased.

†On leave of absence from Dipartimento di Fisica, Università di Bari, I-70126 Bari, Italy.

${ }^{\ddagger}$ Also at Department of Chemistry and Biomedical Sciences, Kalmar University, S-39182 Kalmar, Sweden.
}

${ }^{\S}$ Corresponding author.

Electronic address: zornoza@icecube.wisc.edu

Also at IFIC (CSIC-Universitat de València), AC 22085, 46071 Valencia, Spain.

[1] C. Barat et al., Astron. Astrophys. 79, L24 (1979); T. Cline et al., International Astronomical Union Circular No. 7002 (1998).

[2] P. M. Woods et al., astro-ph/0602402 [Astrophys. J. (to be published)].

[3] D. Borkowski et al., GRBs Coordinates Network Circular No. 2920 (2004); S. Mereghetti et al., Astrophys. J. 624, L105 (2005).

[4] D. M. Palmer et al., GRBs Coordinates Network Circular No. 2925 (2004).

[5] K. Hurley et al., Nature (London) 434, 1098 (2005).

[6] S. Corbel et al., Astrophys. J. 478, 624 (1997); S. Corbel and S. S. Eikenberry, Astron. Astrophys. 419, 191 (2004).

[7] N.M. McClure-Griffiths and Bryan M. Gaensler, Astrophys. J. 630, L161 (2005).

[8] C. Thompson and R. C. Duncan, Mon. Not. R. Astron. Soc. 275, 255 (1995).

[9] D. M. Palmer et al., Nature (London) 434, 1107 (2005).

[10] P. Blasi et al., Astrophys. J. 533, L123 (2000); K. Asano and R. Yamazaki, Publ. Astron. Soc. Jpn. 58, 7 (2006).

[11] F. Halzen, H. Landsman, and T. Montaruli, astro-ph/ 0503348 .

[12] J. D. Gelfand et al., Astrophys. J. 634, L89 (2005).

[13] P. B. Cameron et al., Nature (London) 434, 1112 (2005).

[14] K. Ioka et al., Astrophys. J. 633, 1013 (2005).

[15] S. P. Ahlen et al., Astrophys. J. 412, 301 (1993).

[16] M. Drees, F. Halzen, and K. Hikasa, Phys. Rev. D 39, 1310 (1989); F. Halzen, T. Stanev, and G. B. Yodh, Phys. Rev. D 55, 4475 (1997); F. Halzen and D. Hooper, J. Cosmol. Astropart. Phys. 08 (2003) 006.

[17] E. Andrés et al., Astropart. Phys. 13, 1 (2000).

[18] M. Stamatikos, J. Kurtzweil, and M. J. Clarke, astro-ph/ 0510336; K. Kuehn, AIP Conf. Proc. 836, 616 (2006).

[19] G. C. Hill, J. Hodges, B. Hughey, A. Karle, and M. Stamatikos, in Proceedings of PHYSTAT, Oxford, 2005 (Imperial College Press, Oxford, 2005); G. C. Hill and K. Rawlins, Astropart. Phys. 19, 393 (2003).

[20] D. L. Kaplan et al., Astrophys. J. 556, 399 (2001).

[21] K. Hurley, Nature (London) 397, 41 (1999).

[22] T. Terasawa et al., astro-ph/0502315 [Nature (London) (to be published)].

[23] D. Gotz (private communication).

[24] E. Pian and S. Schwartz (private communication).

[25] D. Heck et al., Forschungszentrum Karlsruhe Report No. FZKA-6019, 1998.

[26] A. Gazizov and M. P. Kowalski, Comput. Phys. Commun. 172, 203 (2005).

[27] J. Conrad et al., Phys. Rev. D 67, 012002 (2003).

[28] I. Moskalenko et al., Astrophys. J. 640, L155 (2006). 